Pacific Journal of Mathematic 


\title{
COBORDISM OF BRANCHED COVERING SPACES
}

\author{
Hugh M. Hilden aNd Robert D. Little
}

We shall develop a general method of constructing branched covering spaces of spheres and other manifolds. In the case of the sphere, this method gives rise via transversality to an equivalence between certain cobordism classes of branched covering spaces and the homotopy groups of certain topological spaces. We will interpret the cobordism equivalence and compute the homotopy groups in one case.

Let $\left(X^{n}, B^{n-2}\right)$ be a pair of finite $\mathrm{CW}$ complexes such that $X^{n}$ is a PL $n$-manifold in a neighborhood of $B^{n-2}$ and $B^{n-2}$ is embedded as a locally flat submanifold. Suppose $\varphi: \pi_{1}\left(X^{n}-B^{n-2}\right) \rightarrow \sum(q)$ is a representation into the permutation group of $q$ letters. (Because we shall identify two such representations if they differ by an inner automorphism of $\Sigma(q)$ there is no need to choose a base point.) Now let $Y^{k}$ be a closed PL manifold and let $f: Y^{k} \rightarrow X^{n}$ be a PL map in general position with respect to $B^{n-2}$. The map $\varphi f_{*}: \pi_{1}\left(Y^{k}-f^{-1}\right.$ $\left.\left(B^{n-2}\right)\right) \rightarrow \Sigma(q)$ is a representation of $\pi_{1}\left(Y^{k}-f^{-1}\left(B^{n-2}\right)\right)$ into $\Sigma(q)$ defined up to an inner automorphism of $\Sigma(q)$. Such an equivalence class of representations defines a $q$-fold branched covering space $p: M^{k}(f) \rightarrow Y^{k}$, branched over the codimension two submanifold $f^{-1}\left(B^{n-2}\right)$ (see [5]). A homotopy $F$ between two such maps $f, g$ : $Y^{k} \rightarrow X^{n}$, that is itself in general position with respect to $B^{n-2}$, gives rise to a cobordism $p: W^{k+1}(F) \rightarrow Y^{k} \times I$, branched over the codimension two submanifold $F^{-1}\left(B^{n-2}\right)$. (We allow branched coverings $p: M^{k}(f) \rightarrow Y^{k}$ where $M^{k}(f)$ is disconnected and we allow the branch set to be empty. Thus, if $f$ is the constant map to a point not on $B^{n-2}$ then $M^{k}(f)$ is $q$ disjoint copies of $Y^{k}$ each mapped to $Y^{k}$ by the identity.)

Two natural questions to ask at this point are:

1. What $q$-fold branched covering spaces $p: M^{k}(f) \rightarrow Y^{k}$ can be obtained in this manner?

2. What cobordisms between $q$-fold branched covering spaces can be obtained?

For at least one set of fixed $X^{n}, B^{n-2}, Y^{k}, q$ and $\varphi$, these questions have geometrically interesting answers. In this case we interpret the cobordism, compute the homotopy group and present the results in the last section.

2. Construction of $X(n)$. Let $A(n)$ denote the subgroup of even permutations of $\Sigma(n)$, the group of permutations of the 
$n$-symbols $\{1, \cdots, n\}$.

Let $G(n)$ be the pullback of $\Sigma(n)$ and $Z$ over $Z / 2 Z$. That is $G(n)=\left\{\left(g_{1}, g_{2}\right) \in \Sigma(n) \oplus Z \mid \alpha\left(g_{1}\right)=\beta\left(g_{2}\right)\right\} \quad$ where $\alpha: \Sigma(n) \rightarrow Z / 2 Z$ and $\beta: Z \rightarrow Z / 2 Z$ are the natural maps.

The group $G(n)$ has the universal property that the homomorphism indicated by the dotted line exists provided all the solid line homomorphisms exist in the commutative diagram below where $\alpha, \beta, \gamma$, and $\delta$ are all natural maps.

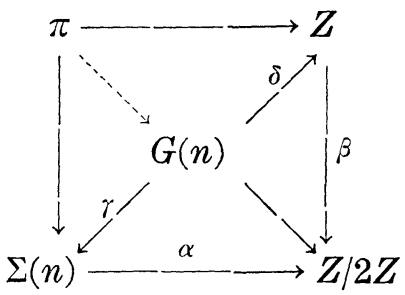

LEMMA 1. Given any $n$ and $k$, there is a connected PL manifold $\hat{X}(n)$ with the following properties:

(a) $\pi_{j}(\hat{X}(n))=0,2 \leqq j \leqq k$.

(b) $\pi_{1}(\hat{X}(n))=G(n)$.

(c) There is a manifold $K(n)$, with $\pi_{1}(K(n))=A(n), \pi_{j}(K(n))=$ $0,2 \leqq j \leqq k$ and a fibration $K(n) \rightarrow \hat{X}(n) \rightarrow S^{1}$.

Proof. Pick $q>k+2$ and let $E(n)$ be the $n$-fold cartesian product of $E^{q}$ with the big diagonal deleted (i.e., those elements with at least one pair of identical coordinates). We can prove inductively that $E(n)$ is $k$ connected by studying the fibration, $E^{q}-(n-1$ points $) \rightarrow E(n) \rightarrow E(n-1)$, obtained by projecting on the first $n-1$ coordinates.

We have natural actions of $A(n)$ and $\Sigma(n)$ on $E(n)$ by permuting coordinates. Let $K(n)$ and $L(n)$ be the respective quotient spaces and let $\tau: K(n) \rightarrow K(n)$ be the covering transformation map of the double covering $K(n) \rightarrow L(n)$. Let $\hat{X}(n)=K(n) \times[0,1] /(x, 0)=$ $(\tau(x), 1)$. We have a fibration $K(n) \rightarrow \hat{X}(n) \rightarrow S^{1}$ and properties $a$ and $c$ easily follow. It remains to be shown that $\pi_{1}(\hat{X}(n))=G(n)$.

The space $\hat{X}(n)$ is double covered by $Y(n)=K(n) \times[0,2] /(x, 0)=$ $(x, 2)$ with projection $(x, t) \rightarrow(x, t)$ if $0 \leqq t<1$ and $(x, t) \rightarrow(\tau(x)$, $t-1)$ if $1 \leqq t<2$. The space $Y(n)$ is covered by $E(n) \times R^{1}$ with projection $\left(\left(x_{1}, \cdots, x_{n}\right), t\right) \rightarrow\left(p\left(x_{1}, \cdots, x_{n}\right), t(\bmod 2)\right)$ where $p: E(n) \rightarrow$ $K(n)=E(n) / A(n)$ is natural.

Now fix a base point $\left(\left(x_{1}, \cdots, x_{n}\right), 0\right)$ in $E(n) \times R^{1}$ and let $\widehat{x}_{0}$ be the image of that base point under the covering space map $E(n) \times$ $R^{1} \rightarrow \hat{X}(n)$. If $[g] \in \pi_{1}\left(\hat{X}(n), \widehat{x}_{0}\right)$, let $\left(\left(y_{1}, \cdots, y_{n}\right), m\right)$ be the endpoint of the lift of $g$ that begins at $\left(\left(x_{1}, \cdots, x_{n}\right), 0\right)$. There are maps 
$\theta: \pi_{1}\left(\hat{X}(n), \hat{x}_{0}\right) \rightarrow \Sigma(n)$ defined by taking $[g]$ into the coordinate permutation that sends $\left(x_{1}, \cdots, x_{n}\right)$ to $\left(y_{1}, \cdots, y_{n}\right)$, and $\psi: \pi_{1}\left(\hat{X}(n), \widehat{x}_{0}\right) \rightarrow Z$, defined by taking $[g]$ into $m$. It can be checked directly from the definitions that $\alpha \theta=\beta \psi$ and that an isomorphism is induced from $\pi_{1}\left(\hat{X}(n), \hat{x}_{0}\right)$ onto $G(n)$.

The manifold $\hat{X}(n)$, depends on $k$, but we will suppress this dependence and just choose $k$ large enough to suit our purposes.

In the remainder of the paper, when we identify $\pi_{1}(\hat{X}(n))$ with $G(n)$ we shall use the base point $\hat{x}_{0}$ and isomorphism $(\theta, \psi)$. Now let $X(n)$ be defined as follows. Let $S^{1}$ be a locally flat circle in $\hat{X}(n)$ representing the element $((1,2), 1)$ in $\pi_{1}\left(\hat{X}(n) ; \hat{x}_{0}\right)$. Then $X(n)=$ $\hat{X}(n) \cup D^{2}$ where the boundary $D^{2}$ is identified with $S^{1}$. Let 0 be the center of $D^{2}$.

LEMMA 2. We have the following computations of homotopy and homology groups

(a) $\pi_{1}(X(n))=0$.

(b) $H_{j}(X(n))=H_{j}(\hat{X}(n)), j \geqq 2$.

Proof. By Van Kampens Theorem $\pi_{1}(X(n))=G(n) /\langle((1,2), 1)\rangle$ where $\langle((1,2), 1)\rangle$ is the normal subgroup generated by $((1,2), 1)$. It can be seen that this group is $G(n)$ using the fact that any two transpositions are conjugate.

The second statement follows from a Mayer-Vietoris argument using the fact that $H_{1}\left(S^{1}\right) \rightarrow H_{1}(\hat{X}(n))$ is an isomorphism because $A(n)$ is the commutator subgroup of $\Sigma(n)$.

We shall adopt the following orientation convention. If $B$ is a codimension two oriented submanifold of the oriented manifold $A$ with normal disc bundle, and $D^{2}$ is a disc fiber of this normal disc bundle, then an orientation of $D^{2}$ is called positive if the orientation of $B$ followed by the orientation of $D^{2}$ gives the orientation of $A$. An orientation of $\partial D^{2}$ is called positive if this orientation followed by the outward normal gives the positive orientation of $D^{2}$.

3. Some technical lemmas and some computations. In this entire section we shall assume that $M^{k-2}$ is a propertly embedded, locally flat not necessarily connected, oriented PL submanifold of the oriented PL manifold $W^{k}$, and that $H_{1}\left(W^{k} ; Z\right)=H_{2}\left(W^{k} ; Z\right)=0$.

Lemma 3. There is a regular neighborhood $U^{k}$ of $M^{k-2}$ that is the total space of a linear 2-disc bundle over $M^{k-2}$.

Proof. By [10], there is a block bundle neighborhood of $M^{k-2}$ and there is no obstruction to putting a $D^{2}$ bundle structure on it. 
The projection map $p: U^{k} \rightarrow M^{k-2}$ induces a $D^{2}$ bundle structure on $U^{k} \cap \partial W^{k}$ over $\partial M^{k-2}$.

Lemma 4. The group $H_{1}\left(W^{k}-M^{k-2} ; Z\right)$ is free abelian and generated by the meridians of $M^{k-2}$, one generator for each component of $M^{k-2}$. The regular neighborhood $U^{k}$ is the total space of a trivial bundle over $M^{k-2}$.

Proof. Using the exact homology sequence, excision, and a homotopy, we have $H_{1}\left(W^{k}-M^{k-2}\right) \cong H_{2}\left(W^{k}, W^{k}-M^{k-2}\right) \cong H_{2}\left(U^{k}\right.$, $\left.U^{k}-M^{k-2}\right) \cong H_{2}\left(U^{k}, E^{k-1}\right)$ where $E^{k-1}$ is the total space of the $S^{1}$ bundle associated with the linear 2-disc bundle over $M^{k-2}$, as in the preceding lemma. By Poincaré duality and homotopy $H_{2}\left(U^{k}, E^{k-1}\right) \cong$ $H^{k-2}\left(U^{k}, U^{k} \cap \partial W^{k}\right) \cong H^{k-2}\left(M^{k-2}, \partial M^{k-2}\right) \cong$ a free abelian group generated by the components of $M^{k-2}$.

To see that the normal bundle of $M^{k-2}$ is trivial, recall that orientable two plane bundles over a finite complex (in this case $\left.M^{k-2}\right)$ are classified by their Euler class $\left(\chi \in H^{2}\left(M^{k-2}\right)\right.$ ). Since $M^{k-2}$ is properly embedded in $W^{k}$, the Euler class $\chi=i^{*}\left(\varphi i_{*}\left[M^{k-2}\right]\right)$ where $\left[M^{k-2}\right]$ is the fundamental class of $M^{k-2}, i: M^{k-2} \rightarrow W^{k}$ is the inclusion, and $\varphi$ is the Poincaré duality isomorphism for $W^{k}$. Since $H_{2}\left(W^{k}\right)=$ $H_{1}\left(W^{k}\right)=0$, we have $H^{2}\left(W^{k}\right)=0$ and $\chi=0$.

Alternatively, represent the element $(+1,+1, \cdots,+1)$ in $H^{1}\left(W^{k}-U^{k} ; Z\right)$ by a map $f: W^{k}-U^{k} \rightarrow S^{1}$, put $f$ in general position and pullback a point to get an orientable "Seifert surface" $F^{k-1}$ with $\partial F^{k-1}=M^{k-2}$. The outward normal gives a cross-section of the associated $S^{1}$ bundle so $D^{2} \rightarrow U^{k} \rightarrow M^{k-2}$ is trivial.

Lemma 5. Let $\rho: \pi_{1}\left(W^{k}-M^{k-2}\right) \rightarrow \Sigma(n)$ be a representation in which meridians are mapped to transpositions. There is a unique representation (up to an inner automorphism of $G(n)) \sigma: \pi_{1}\left(W^{k}\right.$ $\left.M^{k-2}\right) \rightarrow G(n)$ such that $\gamma \sigma=\rho$ and positive meridians are mapped into +1 by $\beta \sigma$.

Proof. This follows immediately from Lemma 4 and the properties of $G(n)$ as pullback of $\Sigma(n)$ and $Z$ over $Z / 2 Z$.

At this point, to obtain further results we must specialize to the case $n=3$.

Proposition 6. Let $M^{k-2}$ be an oriented (not necessarily connected) properly embedded, submanifold of the compact oriented manifold $W^{k}$. Suppose $H_{1}\left(W^{k}\right)=H_{2}\left(W^{k}\right)=0$ and let $\rho: \pi_{1}\left(W^{k}\right.$ $\left.M^{k-2}\right) \rightarrow \Sigma(3)$ be a representation in which meridians are sent to transpositions. 
There is a map $f: W^{k} \rightarrow X(3)$, in general position with respect to the point 0 , with the following properties:

(a) $f^{-1}(0)=M^{k-2}$.

(b) The composition map $\gamma\left(f \mid W^{k}-M^{k-2}\right)_{*}: \pi_{1}\left(W^{k}-M^{k-2}\right) \rightarrow$ $\pi_{1}(X(3)-0) \rightarrow G(3) \rightarrow \Sigma(3)$ is equivalent to $\rho$, where equivalence means equivalence up to an inner automorphism of $\Sigma(3)$.

(c) The induced map $H_{1}\left(W^{k}-M^{k-2}\right) \rightarrow H_{1}(X(3)-0) \rightarrow Z$ sends each positive meridian to +1 .

(d) If $g$ is another such map satisfying $a, b$, and $c$, then $g \cong f$.

Proof. By Lemma $3 U^{k}=M^{k-2} \times D^{2}$. Let $E^{k-1}$ be $M^{k-2} \times S^{1}$.

By Lemma $4 H_{1}\left(W^{k}-M^{k-2}\right)$ is a free abelian group generated by one positive meridian for each component of $M^{k-2}$. (Since $M^{k-2}$ and $W^{k}$ are both oriented, it makes sense to define positive meridians.)

By Lemma 5 there is a unique representation $\sigma: \pi_{1}\left(W^{k}-M^{k-2}\right) \rightarrow$ $G(3)$ such that $\gamma \sigma=\rho$ and positive meridians are mapped into +1 by $\beta \sigma$. Define a function $f_{1}: \overline{W^{k}-U^{k}} \rightarrow X(3)-0$ such that $f_{1 *}: \pi_{1}\left(\overline{W^{k}-U^{k}}\right) \cong \pi_{1}\left(W^{k}-M^{k-2}\right) \rightarrow \pi_{1}(X(3)-0) \rightarrow G(3)$ is equivalent to $\sigma$. This is done by defining $f_{1}$ on the 1-skeleton by using the definition of $\sigma$ on the generators, extending to the 2-skeleton using the fact that $\sigma$ is a homomorphism, and extending to the rest of $W^{k}-U^{k}$ using $\pi_{j}(X(3)-0)=0,2 \leqq j \leqq k$. (See Lemma 1.)

Now let $E_{a}^{k-1}$ be a component of $E^{k-1}$, let $S_{a}^{1}$ be some fiber and let $s_{0} \in S_{a}^{1}$. We wish to investigate $\left[E_{u}^{k-1}, X(3)-0\right]$. First notice that if $f$ and $g \in\left[E_{a}^{k-1}, X(3)-0\right]$ then $f$ and $g$ are homotopic if and only if their restrictions to the 1-skeleton are homotopic since $\pi_{j}(X(3)-0)=0,2 \leqq j \leqq k$. Also, their restrictions to the 1-skeleton are homotopic if and only if they induce the same homomorphism $\pi_{1}\left(E_{a}^{k-1}\right) \rightarrow \pi_{1}(X(3)-0)=G(3)$ up to an inner automorphism of $\pi_{1}(X(3)-0)$. Next we restrict our attention to the subset of $\left[E_{a}^{k-1}, X(3)-0\right]^{\prime}$ consisting of the functions that take the positive meridian $\left[S_{a}^{1}\right]$ to +1 in homology. We denote this subset by $\left[E_{a}^{k-1}, X(3)-0\right]^{\prime}$. Since $E_{a}^{k-1}=M_{a}^{k-2} \times S^{1}$, the homotopy class $x$ of the positive meridian $S_{a}^{1}$ is central in $\pi_{1}\left(E_{a}^{1}, s_{0}\right)$. If $y$ is any element of $\pi_{1}\left(E_{a}^{1}, s_{0}\right)$ and $f \in\left[E_{a}^{k-1}, X(3)-0\right]$, then $f_{*}(y)$ commutes with $f_{*}(x)$. If aslo $f \in\left[E_{a}^{-1}, X(3)-0\right]^{\prime}$, then $f_{*}(x)=((a, b), 1)$ for some transposition $(a, b)$ in $\Sigma(3)$. If we check the commutativity relations in $G(3)$ and $\Sigma(3)$. (It is exactly at this point we need $n=3$.) We see that $f_{*}(y)$ commutes with $f_{*}(x)$ implies that $f_{*}(y)$ is a power of $f_{*}(x)$. Thus if $f$ belongs to $\left[E_{a}^{k-1}, X(3)-0\right]^{\prime}$, it follows that $f_{*} \pi_{1}\left(E_{a}^{k-1}\right)$ is the infinite cyclic subgroup of $G(3)$ generated by $((a, b), 1)$ and it also follows that two elements $f, h$ of $\left[E_{a}^{k-1}, X(3)-0\right]^{\prime}$ are homotopic 
if and only if they induce the same homomorphism; $H_{1}\left(E_{a}^{k-1}\right) \rightarrow$ $H_{1}(X(3)-0)=Z$.

Recall that $X(3)=\hat{X}(3) \cup s^{1} D^{2}$. The first homology of $\hat{X}(3)$ is carried by $S^{1}$ and $X(3)-0 \cong \hat{X}(3)$. Let $h: E_{a}^{k-1} \rightarrow S^{1}$ be such that $h$ (composed with the inclusion $S^{1} \rightarrow X(3)-0$ ) induces the same homomorphism on homology as $f_{1} \mid E_{a}^{k-1}$. We can also assume (by simultaneously homotoping the "lifts" to $R^{1}$ of the maps on the fibers to $\left.S^{1}\right)$ that $h$ maps each fiber homeomorphically onto $S^{1}$. By the preceding remarks $f_{1} \mid E_{a}^{k-1}$ is homotopic to $h$. Thus by homotoping $f_{1}$ near each component of $E^{k-1}$ we may assume that $f_{1}$ maps $\left(W^{k}-U^{k}\right) \cup E^{k-1}$ into $X(3)-0$ in such a way that each fiber of the PL bundle $S^{1} \rightarrow E^{k-1} \rightarrow M^{k-2}$ is mapped homeomorphically onto $S^{1}$. We can now define the sought after map $f: W^{k} \rightarrow X(3)$ by simultaneously coning in each fiber onto the disc $D^{2}$. We are done with parts $a, b, c$.

If $g$ is another map satisfying $a, b$, and $c$, then we can homotope $g$ so that $g$ and $f$ are both fiber maps to the trivial bundle neighborhood of 0 . Restricting $f$ and $g$ to $E^{k-1}$, we see by $b$ and $c$ that they induce the same homomorphism $H_{1}\left(E^{k-1}\right) \rightarrow H_{1}(X(3)-0)$ and since $H_{1}\left(S^{1}\right) \rightarrow H_{1}(X(3)-0)$ is an isomorphism the restrictions induce the same homomorphism $H_{1}\left(E^{k-1}\right) \rightarrow S^{1}$. Thus the restrictions are homotopic, so with no loss of generality we can assume $g=f$ on $U^{k}$.

By $b$ and $c$ and $\pi_{j}(\hat{X}(3))=0,2 \leqq j \leqq k$, we have $g \mid\left(W^{k}-U^{k}\right) \cong$ $f\}\left(W^{k}-U^{k}\right)$. The homotopy $f_{t}$ restricted to $F_{a}^{k-1}=M^{k-2} \times S^{1}$ gives a map $F: E_{a}^{k-1} \times S^{1} \rightarrow X(3)-0$ by $F(x, t)=f_{t}(x), 0 \leqq t \leqq 1$. Since $\pi_{1}\left(E_{a}^{k-1} \times S^{1}\right) \cong \pi_{1}\left(E_{a}^{k-1}\right) \oplus \pi_{1}\left(S^{1}\right)$ and $F_{*}\left(\pi_{1}\left(E_{a}^{k-1}\right) \oplus\{0\}\right)$ is infinice cyclic generated by $((a, b), 1)$, the same commutativity argument we gave before shows $F_{*}\left(\pi_{1}\left(E_{a}^{k-1} \times S^{1}\right)\right)$ is infinite cyclic generated by $((a, b)$, 1). As before an investigation of $\left[E_{a}^{k-1} \times S^{1} ; X(3)-0\right]$ shows we can homotope the homotopy so that $f_{*}\left(E_{a}^{k-1}\right)$ takes values in $S^{1}$. Thus we can extend the homotopy to a homotopy from $f$ to $g$ and we are done.

4. Computation of homotopy groups. The purpose of this section is to compute the first two nontrivial homotopy groups of $X(3)$. We begin with a lemma in which the integral homology and cohomology groups of $X(3)$ are computed. Note that since $X(3)$ is simply connected (Lemma 2), we have $H^{1}(X(3) ; Z)=0$ and so $i=1$ is an exceptional value which must be excluded from the table of values for $H^{*}(X(3) ; Z)$.

LemMa 7. For $i>0$, we have: 
(a)

$$
\begin{aligned}
& H_{i}(X(3) ; Z)=\left\{\begin{array}{l}
0, i \equiv 1,2(\bmod 4) \\
Z_{3}, i \equiv 0,3(\bmod 4)
\end{array}\right. \\
& H^{i}(X(3) ; Z)=\left\{\begin{array}{l}
0, i \equiv 2,3(\bmod 4) \\
Z_{3}, i \equiv 0,1(\bmod 4)
\end{array}\right.
\end{aligned}
$$

Proof. It is clear from Lemma 2 and the universal coefficient theorem that it is sufficient to prove the above assertions for the homology groups of $\hat{X}(3)$. In Lemma 1, we saw that we have a fibration $K(3) \rightarrow \hat{X}(3) \rightarrow S^{1}$, where $K(3)$ is a $K\left(Z_{3}, 1\right)$, since it is the orbit space of a free action of $Z_{3}$. The Serre homology spectral sequence of this fibration converges to $H_{*}(\hat{X}(3) ; Z)$ with $E^{2}$-term given by $E_{p, q}^{2}=H_{p}\left(S^{1} ; H_{q}\left(Z_{3}, 1 ; Z\right)\right)$. Here we mean cohomology with local coefficients in the local system of groups, $H_{*}\left(Z_{3}, 1 ; Z\right)$, given by the action of $\pi_{1}\left(S^{1}\right)$.

The group $\pi_{1}\left(S^{1}\right)$ acts nontrivially on $H_{1}\left(Z_{3}, 1 ; Z\right)$, for otherwise the short exact sequence of fundamental groups given by the fibration would split. It follows from the universal coefficient theorem that $\pi_{1}\left(S^{1}\right)$ acts nontrivially on $H^{2}\left(Z_{3}, 1 ; Z\right)$. Since $H^{*}\left(Z_{3}, 1 ; Z\right)$ is a polynomial algebra on a generator of degree 2 , it follows that the action of $\pi_{1}\left(S^{1}\right)$ is nontrivial in dimensions congruent to $2 \bmod 4$ and trivial in dimensions congruent to $0 \bmod 4$. Using the universal coefficient theorem again, we can infer that the action of $\pi_{1}\left(S^{1}\right)$ on $H_{*}\left(Z_{3}, 1 ; Z\right)$ is nontrivial in dimensions congruent to $1 \bmod 4$ and trivial in dimensions congruent to $3 \bmod 4$. With this information it is easy to compute the boundary operator for the singular chains of $S^{1}$ with coefficients in the local system $H_{*}\left(Z_{3}, 1 ; Z\right)$ and conclude that: $E_{p, q}^{2}=Z_{3}, p=0$ or $1, q \equiv 3(\bmod 4)$, and $E_{p, q}^{2}=0$ otherwise. It follows that the homology groups of $\hat{X}(3)$ are exactly as stated in the lemma.

Proposition 8. The space $X(3)$ is 2-connected and:
(a) $\pi_{3}(X(3))=Z_{3}$.
(b) $\pi_{4}(X(3))=Z_{3}$.
(c) $\pi_{5}(X(3))=0$.

Proof. The facts that $X(3)$ is 2-connected and that $\pi_{3}(X(3))=Z_{3}$ follow immediately from Lemmas 2 and 7 together with the Hurewicz theorem. To prove the second two statements of the theorem, observe that by Lemma 7 and the universal coefficient theorem, there exists a map $f: X(3) \rightarrow K\left(Z_{3}, 3\right) \times K\left(Z_{3}, 4\right)$ which induces integral homology isomorphisms in dimensions less than or equal to 4 . But the known computations of the homology of Eilenberg-MacLane spaces in [4] and [3], together with the Künneth formula show that 
the integral homology of $K\left(Z_{3}, 3\right) \times K\left(Z_{3}, 4\right)$ is zero in dimensions 5 and 6 . Therefore, by Lemma 7 and the Whitehead theorem, $f$ induces an isomorphism of homotopy groups in dimensions less than or equal to 5 and the proof of the proposition is complete.

The authors would like to thank the referee for suggesting the above proof of Proposition 8. It is shorter and simpler that the author's original argument.

5. Interpretation of results and remarks. An $n$ to one branched covering $p: M^{m} \rightarrow N^{n}$ is simple if for each point $x \in N^{n}, p^{-1}(x)$ contains $n-1$ or $n$ points. Berstein and Edmonds studied simple branched coverings in [1] where they proved, among other things, that the simple branched coverings $p: M^{3} \rightarrow S^{3}$, of given degree are an open dense set in the set of all branched coverings $p: M^{3} \rightarrow S^{3}$, of given degree. It is a result of the first author [6] and José Montesinos [8] that every closed orientable 3-manifold is a three to one simple branched covering of $S^{1}$ branched over a 1-manifold (a knot or link). Montesinos has shown [7] that every closed orientable 4-manifold $W^{4}$ has a handlebody decomposition, $W^{4}=H^{0} \cup \lambda H^{1} \cup$ " $H^{2} \cup \gamma H^{3} \cup H^{4}$, such that $W^{4}$ is uniquely determined by $H^{0} \cup \lambda H^{1} \cup$ $\mu H^{2}$ and $H^{0} \cup \lambda H^{1} \cup \mu H^{2}$ is a 3 -fold simple branched covering of $D^{4}$, branched over a properly embedded but not necessarily orientable surface.

Berstein and Edmonds showed in [2] that there are 4-manifolds, including $S^{1} \times S^{\prime} \times S^{1} \times S^{1}$, that are not 3-fold branched coverings of $S^{4}$. It is not known just what closed orientable 4-monifolds are 3 -fold simple branched coverings of $S^{4}$ branched over an orientable surface but it is clear that a great many four manifolds have such a representation.

With this as motivation we proceed to study triples $\left(M^{n}, p, B^{n-2}\right)$ where $p: M^{n} \rightarrow S^{n}$ is a $k$ to one simple branched covering space, branched over the oriented closed $n-2$ manifold $B^{n-2}$. We say two such triples $\left(M_{1}^{n}, p_{1}, B_{1}^{n-2}\right)$ and $\left(M_{2}^{n}, p_{2}, B_{2}^{n-2}\right)$ are cobordant if there is a triple $\left(W^{n+1}, q, C^{n-1}\right)$ where $q: W^{n+1} \rightarrow S^{n} \times I$ is a $k$ to one simple branched covering space, branched over the properly embedded oriented submanifold $C^{n-1}$, where $\partial C^{n-1}=B_{1}^{n-2} \cup-B_{2}^{n-2}$, and $M_{1}^{n}=$ $q^{-1}\left(S^{n} \times\{0\}\right), M_{2}^{n}=q^{-1}\left(S^{n} \times\{1\}\right)$, and $q \mid M_{1}^{n}=p_{1}$ and $q \mid M_{2}^{n}=p_{2}$.

Next, we orient the disc $D^{2}$ in $X(k)$ so that $\partial D^{2} \rightarrow+1$ under the $\operatorname{map} H_{1}(X(k)-0) \rightarrow Z$.

For each $n$ and $k$ there is a map $\theta(n, k)$ from $\pi_{n}(X(k))$ to cobordism classes of triples defined as follows: Orient $S^{n}$ and consider $f: S^{n} \rightarrow X(k)$. Throw $f$ in general position with respect to 0 and let $B^{n-2}=f^{-1}(0)$. Then $B^{n-2}$ has a neighborhood $U^{n}$ mapped onto a smaller disc $D^{2 \prime} \subset D^{2}$ as a linear 2-disc bundle. Orient $B^{n-2}$ and the 
fibres in $U^{n}$ so that the product of the orientations gives the orientation of $S^{n}$ and so that the boundaries of the fibers are mapped into +1 by $H_{1}\left(\overline{S^{n}-U^{n}}\right) \rightarrow H_{1}(X(k)-0) \rightarrow Z$. Let $p: M^{n} \rightarrow S^{n}$ be the $k$ to one simple branched covering defined by the equivalence class of representations $\pi_{1}\left(S^{n}-B^{n-2}\right) \rightarrow \pi_{1}(X(k)-0) \rightarrow \pi_{1}(\hat{X}(k))=G(k) \rightarrow$ $\Sigma(k)$. Then $\theta(n, k):[f] \rightarrow\left(M^{n}, p, B^{n-2}\right)$. It is easy to see that $\theta(n, k)$ is well defined by throwing any homotopy in general position with respect to 0 . At this point we specialize to $k=3$.

It follows immediately from Proposition 6, (and the $1-1$ correspondence between simple branched coverings of $S^{n}$ branched over $B^{n-2}$ and equivalence classes of representations $\pi_{1}\left(S^{n}-B^{n-2}\right) \rightarrow \Sigma(k)$ in which meridians are sent to transpositions) that $\theta(n, 3)$ is onto for $n \geqq 3$.

It also follows from part $d$, of Proposition 6 that $\theta(n, 3)$ is $1-1$.

We summarize this in a theorem that concludes the paper.

THEOREM 9. If $n=3$ or $n=4$, there are exactly three cobordism classes of 3-fold simple branched covering spaces, $p: M^{n} \rightarrow S^{n}$, branched over oriented submanifolds, if $n=5$ there is only one.

It may be useful, when $n=3$, to have representatives for each of the three classes of Theorem 9. Let $f: S^{3} \rightarrow X(3)$ be in general position with respect to 0 so that $f^{-1}(0)$ is a knot. We have the following commutative diagram of "double branched coverings."

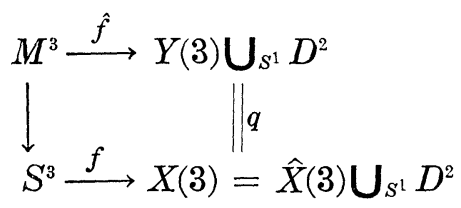

Here $p: M^{3} \rightarrow S^{3}$ is the usual double branched covering of the knot $k=f^{-1}(0)$ and $Y(3)=K(3) \times S^{1}$ is defined in the proof of Lemma 1 . The map $q: Y(3) \rightarrow \hat{X}(3)$ is the double (unbranched) covering map and $q: D^{2} \rightarrow D^{2}$ is the usual double branched covering branched over 0 . We can choose the attaching circle from $D^{2}$ to $Y(3)=K(3) \times S^{1}$ to be of the form $* \times S^{1}$. We note that $\pi_{j}(Y(3))=0,2 \leqq j \leqq 4$ and $\pi_{1}(Y(3))=Z_{3} \oplus Z$. The question is, when does $f: S^{3} \rightarrow X(3)$ extend to $D^{4}$ ?

By the Hurewicz theorem, the map $f$ extends if and only if $f_{*}(x)=0$ where $x \in H_{3}\left(S^{3} ; Z\right)$ is a generator (see Section 8). But $f_{*}(x)=0$ if and only if $\hat{f}_{*}(y)=0$ where $y \in H_{3}\left(M^{3} ; Z\right)$ is a generator. This is so because $p_{*}: H_{3}\left(M^{3} ; Z\right) \rightarrow H_{3}\left(S^{3} ; Z\right)$ is multiplication by two and $\left.q_{*}: H_{3}\left(Y^{3} \cup_{S^{1}} D^{2}\right) ; Z\right)=H_{3}\left(Y^{3} ; Z\right)=Z_{3} \rightarrow H_{3}\left(\hat{X}(3) \cup_{S^{1}} D^{2}\right)=H_{3}(\hat{X}(3))=Z_{3}$ is an isomorphism. The natural "projection" map $\pi: Y(3) \cup D^{2}=$ 
$K(3) \times S^{1} \cup D^{2} \rightarrow K(3)$ sends $* \times S^{1} \cup D^{2}$ into $*$ and also induces an isomorphism on $H_{3}(; Z)$. Thus we have a map $\pi \hat{f}:\left(M^{3}, U\right) \rightarrow\left(Y(3) \cup_{S^{1}}\right.$ $\left.D^{2}, D^{2}\right) \rightarrow(K(3), *)$. where $U$ is a regular neighborhood of the branch cover $p^{-1}(k)$. We now have that $f$ extends if and only if $(\pi \hat{f})_{*}(y)=0$. At this point we investigate the homotopy classes of maps $\left[M^{3}, U ; K(3), *\right]$.

Obstruction theory tells us this group is the same as $H^{1}\left(M^{3}, U ; Z_{3}\right)$. We also have $0 \rightarrow H^{1}\left(M^{3}, U ; Z_{3}\right) \rightarrow H^{1}\left(M^{3} ; Z_{3}\right) \rightarrow$ $H^{1}\left(U ; Z_{3}\right)$ from the long cohomology sequence and $H^{1}\left(M^{3} ; Z_{3}\right) \rightarrow$ $H^{1}\left(U ; Z_{3}\right)$ is the zero map because the preimage of the branch set bounds either one of the two lifts of any Seifert surface for $k$. Thus $H^{1}\left(M^{3}, U ; Z_{3}\right) \cong H^{1}\left(M^{3} ; Z_{3}\right)$ and we get the following result.

THEOREM 10. Let $p: M^{3} \rightarrow S^{3}$ be a three-fold simple branched covering, branched over the knot $k$. If the double branched covering of $k$ has trivial first homology with $Z_{3}$ coefficients, then $p: M^{3} \rightarrow S^{3}$ extends to $p: W^{4} \rightarrow D^{4}$ branched over an orientable surface.

Now suppose that $\pi_{1}\left(M^{3}\right)=Z_{3}$ and $\pi_{2}\left(M^{3}\right)=0$ such as for the Lens space $L(3,1)$. We wish to show that $(\pi \hat{f})_{*}(y) \neq 0$ in $H_{3}(K(3)$, $* ; Z)=Z_{3}$ where $y$ generates $H_{3}\left(M^{3}, U ; Z\right)$. It is enough to show $(\pi \hat{f})_{*}(w) \neq 0$ in $H_{3}(K(3) ; Z)$ where $w$ generates $H_{3}\left(M^{3} ; Z\right)$. We know that $\pi \hat{f}$ maps $\pi_{1}\left(M^{3}\right)$ onto $\pi_{1}(K(3))$ and is therefore an isomorphism. (Recall $\pi_{1}\left(M^{3}-p^{-1}(k)\right)$ is mapped onto $\pi_{1}(Y(3))=Z \oplus Z / 3$ by the representation.) For purposes of computation we replace the map $\pi \hat{f}: M^{3} \rightarrow K(3)$ by an inclusion map $M^{3} \hookrightarrow K(3)$. From the homotopy sequence of the pair, we conclude that $\pi_{j}\left(K(3), M^{3}\right)=0 j=1,2,3$. By the Hurewicz theorem $H_{j}\left(K(3), M^{3}\right)=0$ if $j=1$, 2, or 3 . Thus $\pi \hat{f}: H_{3}\left(M^{3}\right) \rightarrow H_{3}(K(3))$ is surjective and $f: S^{3} \rightarrow X(3)$ does not extend to $D^{4}$.

We observe (see [9]) that the double branched covering of the trefoil knot is the Lens space $L(3,1)$ and a reflection in $S^{3}$ sends the left-hand trefoil knot to the right-hand trefoil. The last theorem now follows easily.

THEOREM 11. The three cobordism classes of oriented simply represented knots in $S^{3}$ are represented by the left-and right-hand trefoil knots with their usual representations and any simply represented knot (such as a slice knot) whose double branched covering space has trivial first homology.

The authors would like to again express their appreciation to the referee and to José Montesinos for their helpful observations. 
Added in proof. Recently, extensive calculations of some of the groups defined in $\S 1$ have been made by Neal Brand and Gregory Brumfiel. Also Neal Brand has defined a classifying space for branched coverings.

\section{REFERENCES}

1. I. Berstein and A. Edmonds, On the construction of branched coverings of low dimensional manifolds, (preprint).

2. - The degree and branch set of a branched covering.

3. H. Cartan, Sur les groupes d' Eilenberg-MacLane II, Proc. at. Acad. Sci. U. S. A., 40 (1954), 704-707.

4. S. Eilenberg and S. MacLane, On the groups of $H(I, n) I$. Ann. of Math., (2) 58 (1953), 55-106.

5. R. H. Fox, Quick Trip through Knot Theory, in Topology of 3-manifolds and Related Topics, Ed. M. K. Fort Jr., Prentice-Hall, Inc.

6. H. Hilden, Three-fold branched coverings of $S^{3}$, Amer. J. Math., 98 (1976), 989-997.

7. J. M. Montesinos, 4-manifolds, 3-fold coverings spaces and ribbons, (preprint).

8. - Three-manifolds as 3-fold branched covers of $S^{3}$, Quart. J. Math. Oxford Ser., 27 (1976).

9. Dale Rolfsen, Knots and Links, Pulish or Perish press.

10. C. P. Rourke and B. J. Sanderson, Block bundles I, Ann. of Math., 87 (1968), 1-28.

Received May 28, 1978 and in revised form September 20, 1978. The first author's reseach was supported by a National Science Foundation grant.

UNIVERSITY OF HAWAII

HONOLULU, HI 96822 

.././. ./FrontMatter/paper .pdf 


\section{Pacific Journal of Mathematics \\ Vol. 87, No. $2 \quad$ February, 1980}

Theagenis Abatzoglou, Unique best approximation from a $C^{2}$-manifold in Hilbert space ................................. 233

Gerald Arthur Anderson, $\Lambda$-homology cobordism bundles............. 245

Eric Bedford, Holomorphic mapping of products of annuli in $\mathbf{C}^{n} \ldots \ldots \ldots 271$

Gunnar Carlsson, On the stable splitting of $b o \wedge b o$ and torsion operations in connective $K$-theory .......................... 283

Lester Eli Dubins and David Samuel McIntyre Margolies, Naturally integrable functions ................................. 299

Leo Egghe, The Radon-Nikodým property, $\sigma$-dentability and martingales in locally convex spaces ............................. 313

Irving Leonard Glicksberg, Maps preserving translates of a function ..... 323

Hugh M. Hilden and Robert D. Little, Cobordism of branched covering

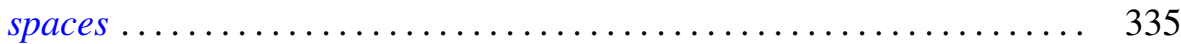

Russell Allan Johnson, Almost-periodic functions with unbounded integral .......................................... 347

Bruce Stephen Lund, The endomorphisms of a Dirichlet algebra ........ 363

John Henry McCleary, Mod $p$ decompositions of H-spaces; another approach........................................ 373

Arlan Bruce Ramsay, Subobjects of virtual groups ................ 389

Thomas R. Savage, Generalized inverses in regular rings ............ 455 Jaak Vilms, On curvature operators of bounded rank ......... 